\title{
Trauma-Induced Cutaneous Focal Mucinosis of the Mammary Areola: An Unusual Presentation
}

\author{
Werner Kempf ${ }^{a} \quad$ Britta von Stumberg ${ }^{b} \quad$ Natalja Denisjuk ${ }^{a} \quad$ Beata Bode $^{c}$ \\ Franco Rongioletti ${ }^{d}$ \\ ${ }^{a}$ Kempf und Pfaltz, Histologische Diagnostik, bermatology Practice Haut und Licht, and \\ 'Department of Pathology, Institute of Clinical Pathology, University Hospital Zürich, Zürich, \\ Switzerland; ${ }^{\mathrm{d} D e p a r t m e n t}$ of Health Sciences, DISSAL Section of Dermatology, University of \\ Genoa, Genoa, Italy
}

Key Words

Cutaneous focal mucinosis $\cdot$ Mucinoses $\cdot$ Breast $\cdot$ Trauma

\begin{abstract}
Cutaneous focal mucinosis (CFM) is a localized form of cutaneous dermal mucinosis clinically presenting as an asymptomatic skin-colored papule or nodule. The etiopathogenesis of CFM is unclear, but it is thought to represent a reactive lesion. Although trauma has been suspected as a triggering factor, it has never been proven in cases of CFM. We report 2 male patients with trauma-induced CFM arising at the mammary areola, which is an unusual site for CFM. Both male patients presented with a solitary nodular lesion of up to $2 \mathrm{~cm}$ in diameter at the right areola. Histology was characterized by circumscribed abundant dermal mucin deposits in a polylobulated pattern without an increased number of fibroblasts or capillaries and with absence of an inflammatory infiltrate. Alcian blue stain at $\mathrm{pH} 2.5$ highlighted the mucin deposits. Immunohistochemistry showed partial expression of FXIIIa by $30 \%$ of the stromal cells, but no reactivity for CD34, smooth muscle actin, desmin, CD68 and S-100. A history of trauma (laser-based epilation, piercing) preceded the development of CFM in both patients. Surgical excision resulted in complete remission without recurrence. Follow-up in both our patients did not reveal recurrences. CFM has to be distinguished from benign and malignant myxoid neoplasms.


Kempf et al.: Trauma-Induced Cutaneous Focal Mucinosis of the Mammary Areola: An Unusual Presentation

\section{Introduction}

Cutaneous mucinoses refer to disorders with abnormal accumulation of mucin in the skin in a diffuse or focal pattern [1]. Mucin is an amorphous material that is part of the extracellular matrix with hyaluronic acid as the most important component of dermal mucin. The spectrum of cutaneous mucinoses encompasses hamartomatous-neoplastic and degenerative-inflammatory forms. The latter ones may be associated with systemic disorders such as autoimmune diseases, diabetes mellitus, paraproteinemia or altered thyroid function [2]. Cutaneous focal mucinosis (CFM) is a localized form of cutaneous dermal mucinosis clinically presenting as an asymptomatic skin-colored papule or nodule that occurs anywhere on the body or in the oral cavity. The etiopathogenesis of CFM is unclear, but it is thought to represent a reactive and not a neoplastic lesion. Although trauma has been suspected as a triggering factor, it has never been proven in cases of CFM [3-5]. We report 2 patients with traumainduced CFM arising at an unusual site, i.e. the mammary areola, and discuss the differential diagnoses.

\section{Case Reports}

The first patient was an otherwise healthy 47-year-old man with a solitary, skin-colored nodular lesion of $2 \mathrm{~cm}$ in diameter at the margin of the right areola (fig. 1a). The medical history did not reveal other skin disorders except for a genital wart on the mons pubis. Previous folliculitis had occurred and laser epilation had been performed in the area in which the nodular lesion on the areola developed. Histopathology revealed circumscribed abundant dermal mucin deposits in a polylobulated pattern without an increased number of fibroblasts or capillaries (fig. 2a, b). No inflammatory infiltrate was observed. The overlying epidermis was inconspicuous. Alcian blue stain at $\mathrm{pH} 2.5$ highlighted the mucin deposits (fig. 2c). Immunohistochemistry showed partial expression of FXIIIa by $30 \%$ of the stromal cells, but no reactivity for CD34, smooth muscle- $\alpha$ actin (SMA), desmin, CD68 and S-100. Based on these findings, the lesion was diagnosed as CFM. Hematologic and serologic tests as well as radiologic examination did not show any abnormalities; in particular, there were no signs of thyroid dysfunction, hypergammaglobulinemia or underlying breast carcinoma. The lesion was completely excised. No recurrence was observed during a follow-up period of 9 months.

The second patient was a 30-year-old man who presented with a 3 months' history of a solitary, round, translucent, asymptomatic white exophytic nodule with a bluish hue on the right areola (fig. 1b). The lesion measured $1.2 \mathrm{~cm}$ in diameter. He had worn a nipple ring piercing for 3 years only on the right side. He decided to remove it because of relapsing episodes of infectious dermatitis that had been treated with topical antibiotics. The lesion started a couple of weeks after the ring piercing had been removed with progressive enlargement. The lesion was totally excised. Histopathology showed a polypoid, dome-shaped dermal nodule with abundant mucin filling the dermis, but not extending into the subcutis (fig. 2d). Large cleft-like spaces inside the core were seen. The epidermis was atrophic with a peripheral collarette. Elastic fibers were almost absent. The mucin stained positively with Alcian blue stain at $\mathrm{pH}$ 2.5. Immunohistologically, the stromal cells were positive for vimentin. FXIIIa+ cells accounted for up to $20 \%$ of stromal cells positive for vimentin, and a few dermal dendritic cells were also positive for CD34. The stromal cells were negative for desmin, SMA, CD68 and S-100. Complete blood count, thyroid function tests, serum immunoglobulins, antinuclear antibody, and DNA antibody studies were done to rule out underlying systemic disease, and all results were normal or negative. A diagnosis of CFM was made. 

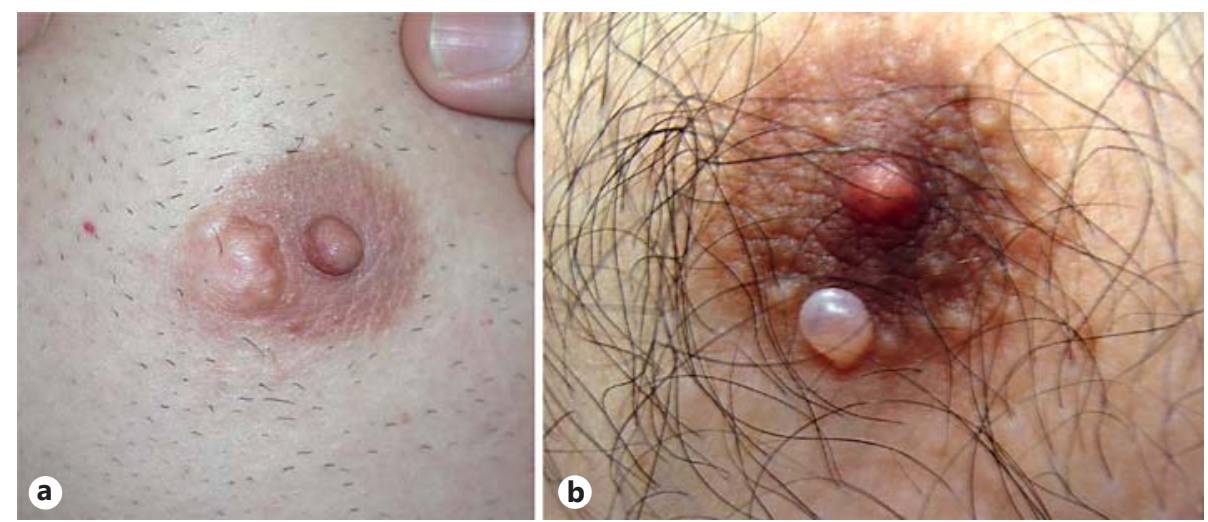

Fig. 1. a A polycyclic nodular lesion on the lateral margin of the right areola mammae (patient 1). b A translucent, asymptomatic white exophytic nodule with a bluish hue on the right areola (patient 2).
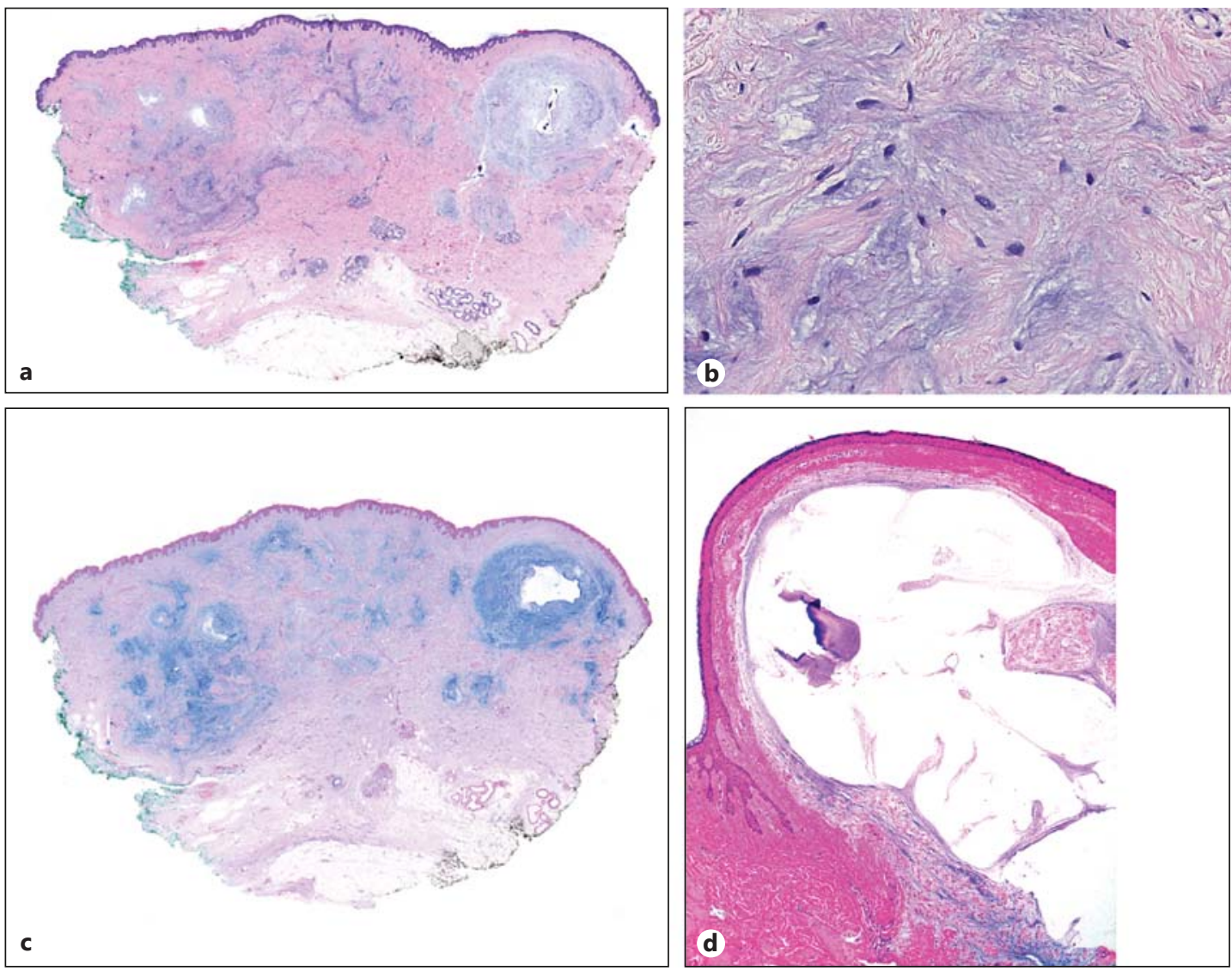

Fig. 2. Dermal ill-defined mucin deposits in patient $1($ HE. $\times 20)$ (a) with inconspicuous number and morphology of fibroblasts (HE. $\times 200$ ) (b). c Mucin deposits stained by Alcian blue at pH 2.5 in patient 1 (Alcian blue. $\times 20$ ). d Polypoid lesion with mucin deposits and pseudocystic features in patient 2 (HE. $\times 20$ ). 
Kempf et al.: Trauma-Induced Cutaneous Focal Mucinosis of the Mammary Areola: An Unusual Presentation

\section{Discussion}

CFM was first described as a separate entity by Johnson and Helwig in 1966 who also defined the histologic criteria for the diagnosis. It is characterized by an asymptomatic, elevated, whitish or skin-colored papule or nodule that is usually solitary and detected on the face, trunk or extremities, but not in proximity of the joints of the hands, wrists or feet. Occasionally, CFM has been reported to occur as a soft fibroma-like polyp or a plaque-like lesion $[4,6]$. CFM occurs in adults of either sex and has also been reported in children [7]. Multiple lesions have rarely been described. Contrary to other forms of primary mucinoses, CFM is usually not associated with systemic manifestations. Jakubovic et al. [18], however, reported a case of multiple CFM with hypothyroidism and myxedema. Moreover, CFM was described in association with other forms of mucinoses such as scleromyxedema and reticular erythematous mucinosis [8]. Interestingly, multiple focal cutaneous mucinoses have been considered as a side effect of anti-TNF- $\alpha$ therapy in a case report [9].

The histology is essential for the diagnosis and shows a diffuse ill-defined dermal accumulation of mucin sparing subcutaneous tissue, with a normal or slightly increased number of fibroblasts and absence of inflammation. Spindle-shaped fibroblasts are the predominant cell type, with occasional admixed factor XIIIa+ dendritic cells. The epidermis may be normal or hyperplastic, sometimes forming a collarette. Elastic fibers are absent and there is no increased vascularity. Follicular induction of the epidermis as in dermatofibroma has also occasionally been described [10].

The cause of CFM is unknown but it is thought to be a reactive lesion arising as a result of the dysfunction of fibroblasts in a circumscribed area. Chronic antigenic stimulation, inflammation, and viral infections could also account for the observed features [11]. Several recent studies have examined the relationship of CD44 receptor expression to dermal hyaluronate mucin accumulation, but trauma has never been explicitly reported as a triggering factor.

In our cases, a history of trauma preceded the development of CFM in both patients. In the first patient, laser-based epilation of hairs and previous folliculitis in the nipple area and in the second patient, piercing might be the relevant traumatic triggers inducing CFM. This form of mucinoses may occur anywhere on the skin, but the mammary areola represents a very unusual localization. To the best of our knowledge, only one case of CFM at the nipple has been reported in the literature [12]. An unusually large size of $2 \mathrm{~cm}$ was seen in our first case while CFM typically measures less than $1 \mathrm{~cm}$ in diameter $[1,2]$.

Differential diagnosis of CFM includes benign and malignant neoplasms with myxoid stroma, such as angiomyxoma, neurothekeoma, nerve sheath myxoma and myxofibrosarcoma. Angiomyxoma (syn. myxoma) is a benign neoplasm, which arises as solitary or multiple lesions. Histologically, a well-circumscribed lesion in the dermis with extension into the subcutis is found, which contains abundant mucin, fibroblasts of variable size and shape, multinucleated histiocytic giant cells as well as an increased number of small capillary vessels [13]. In contrast to CFM, angiomyxoma displays a higher vascularity and expression of SMA by the fibroblastic stromal cells. Whereas CFM is usually less than $1 \mathrm{~cm}$ in diameter and illdefined, angiomyxoma (or myxoma) measures between 1 and $5 \mathrm{~cm}$ in most cases and shows a well-defined, lobulated architecture. The lack of an increased number of vessels as well as thelackofSMA expression arguesagainstangiomyxomain both of our patients. Neurothekeoma is a benign neoplasm with a myxoid variant displaying a lobulated, frequently septated proliferation of fibroblasts embedded in a mucinous stroma [14]. Nerve sheath myxoma is mostly located on the hand and fingers and involves the dermis and/or subcutis. A multilobular tumor mass with abundant myxoid matrix and S-100+ Schwann cells are typically found [15]. Myxofibrosarcoma is a malignant soft tissue neoplasm. Cases arising on the breast have been 
documented [16]. It is characterized by a mucinous tumor stroma and spindle-shaped tumor cells with nuclear pleomorphism and mitotic activity [17]. The cellularity and the phenotype of the tumor cells allow to distinguish CFM from the above-discussed soft tissue tumors. Follow-up in both our patients did not reveal recurrences.

Surgical excision, which is the treatment of choice for CFM, was performed in both of our patients resulting in complete remission without recurrence.

In summary, we reported two cases of trauma-related CFM arising at an unusual site such as the mammary areola. CFM has to be distinguished from benign and malignant neoplasms characterized by mucinous tumor stroma.

\section{References}

1 Rongioletti F, Rebora A: Mucinoses; in Bolognia J, Jorizzo JL, Schaffer JV (eds): Dermatology, ed 3. Philadelphia, Elsevier, 2012.

-2 Rongioletti F, Rebora A: Cutaneous mucinoses: microscopic criteria for diagnosis. Am J Dermatopathol 2001; 23:257-267.

-3 Duperrat B, Vanbremeersch F: Pseudotumoral mucinosis with solitary focus (focal cutaneous mucinosis). Bull Soc Fr Dermatol Syphiligr 1966;73:366-368.

4 Chen HH, et al: A solitary soft fibroma-like polypoid mucinosis: report of an unusual case. Dermatol Surg 2004; 30:450-451.

5 Lee WS, et al: Cutaneous focal mucinosis arising from the chin. J Craniofac Surg 2010;21:1639-1641.

6 Yamamoto M, et al: Plaque-type cutaneous focal mucinosis. Int J Dermatol 2011;50:896-898.

7 Nebrida ML, Tay YK: Cutaneous focal mucinosis: a case report. Pediatr Dermatol 2002;19:33-35.

8 Rongioletti F, et al: Cutaneous focal mucinosis associated with reticular erythematous mucinosis and scleromyxedema. J Am Acad Dermatol 1991;24:656-657.

-9 Duparc A, et al: Multiple lesions of focal cutaneous mucinosis: a side-effect of anti-TNF alpha therapy? Ann Dermatol Venereol 2010;137:140-142.

10 Tatsas AD, et al: Cutaneous focal mucinosis causing follicular induction of the epidermis. Am J Dermatopathol 2009;31:462-464.

11 Wilk M, Schmoeckel C: Cutaneous focal mucinosis - A histopathological and immunohistochemical analysis of 11 cases. J Cutan Pathol 1994;21:446-452.

12 Senff H, et al: Cutaneous myxoma (focal dermal mucinosis). Hautarzt 1988;39:606-610.

13 Wilk M, et al: Cutaneous angiomyxoma: a benign neoplasm distinct from cutaneous focal mucinosis. J Am Acad Dermatol 1995;33:352-355.

14 Fetsch JF, et al: Neurothekeoma: an analysis of 178 tumors with detailed immunohistochemical data and longterm patient follow-up information. Am J Surg Pathol 2007;31:1103-1114.

15 Fetsch JF, Laskin WB, Miettinen M: Nerve sheath myxoma: a clinicopathologic and immunohistochemical analysis of 57 morphologically distinctive, S-100 protein- and GFAP-positive, myxoid peripheral nerve sheath tumors with a predilection for the extremities and a high local recurrence rate. Am J Surg Pathol 2005;29: 1615-1624.

16 Adem C, et al: Primary breast sarcoma: clinicopathologic series from the Mayo Clinic and review of the literature. Br J Cancer 2004;91:237-241.

17 Clarke LE: Fibrous and fibrohistiocytic neoplasms: an update. Dermatol Clin 2012;30:643-656, vi.

18 Jakubovic HR, et al: Multiple cutaneous focal mucinoses with hypothyroidism. Ann Intern Med 1982;96: 56-58. 